\title{
Functional Oligomeric Forms of Uncoupling Protein 2: Strong Evidence for Asymmetry in Protein and Lipid Bilayer Systems
}

\author{
Afshan Ardalan ${ }^{1 \dagger}$, Shahin Sowlati-Hashjin ${ }^{2,3 \dagger}$, Stephanie O. Uwumarenogie ${ }^{1}$, Michael Fish ${ }^{1,4}$, Joel \\ Mitchell1 ${ }^{1}$, Mikko Karttunen²,3,5*, Matthew D. Smith ${ }^{4}$, and Masoud Jelokhani-Niaraki ${ }^{1 *}$ \\ ${ }^{1}$ Department of Chemistry and Biochemistry, Wilfrid Laurier University, Waterloo, ON, Canada N2L 3C5; \\ 2 Department of Chemistry, The University of Western Ontario, London, ON, Canada N6A 3K7; \\ 3 The Center for Advanced Materials and Biomaterials Research, The University of Western Ontario, London, ON, \\ Canada N6K 3K7; \\ ${ }^{4}$ Department of Biology, Wilfrid Laurier University, Waterloo, ON, Canada N2L 3C5; \\ ${ }^{5}$ Department of Applied Mathematics, The University of Western Ontario, London, ON, Canada N6A 5B7
}

\section{Table of Contents}

$\begin{array}{llr}\text { Scheme S1. } & \text { Summary of experimental procedures } & \text { S2 }\end{array}$

$\begin{array}{lll}\text { Table S1. } & \text { Salt-bridges in the oligomeric UCP2 } & \text { S3 }\end{array}$

$\begin{array}{lll}\text { Figure S1. } & \text { Overlay structures } & \text { S3 }\end{array}$

Figure S2. $\quad$ Representation of oligomerization motifs on UCP2 structure $\quad$ S4

$\begin{array}{lll}\text { Figure S3. } & \text { Orientations of UCP2 monomers in dimers } & \text { S4 }\end{array}$

Figure S4. Western blot analysis of AAC1 $\quad$ S5

Figure S5. Backbone RMSD, Rg, and SASA of UCP2 and AAC1 S5

$\begin{array}{lll}\text { Figure S6. } & \text { AAC1 structures } & \text { S6 }\end{array}$

Figure S7. Projection of the MD simulation trajectories on the first 4 eigenvectors $\quad$ S6

$\begin{array}{lll}\text { Figure S8. } & \text { Visualization of principle component analysis } & \text { S7 }\end{array}$

$\begin{array}{llll}\text { Figure S9. } & \text { Initial and final structures for the dissociation process of oligomeric UCP2 } & \text { S7 }\end{array}$

Figure S10. Salt-bridges between the neighboring UCP2 units $\quad$ S8

Figure S11. Density profile of UCP2 and AAC1 $\quad$ S9

$\begin{array}{llr}\text { Figure S12. } & \text { Area per lipid for membranes in UCP2 system } & \text { S10 }\end{array}$

Figure S13. Distribution of lipid headgroups for AAC1 systems $\quad$ S11

$\begin{array}{ll}\text { References } & \text { S11 }\end{array}$

$\dagger^{\dagger}$ Equal contributors.

* Corresponding authors. 


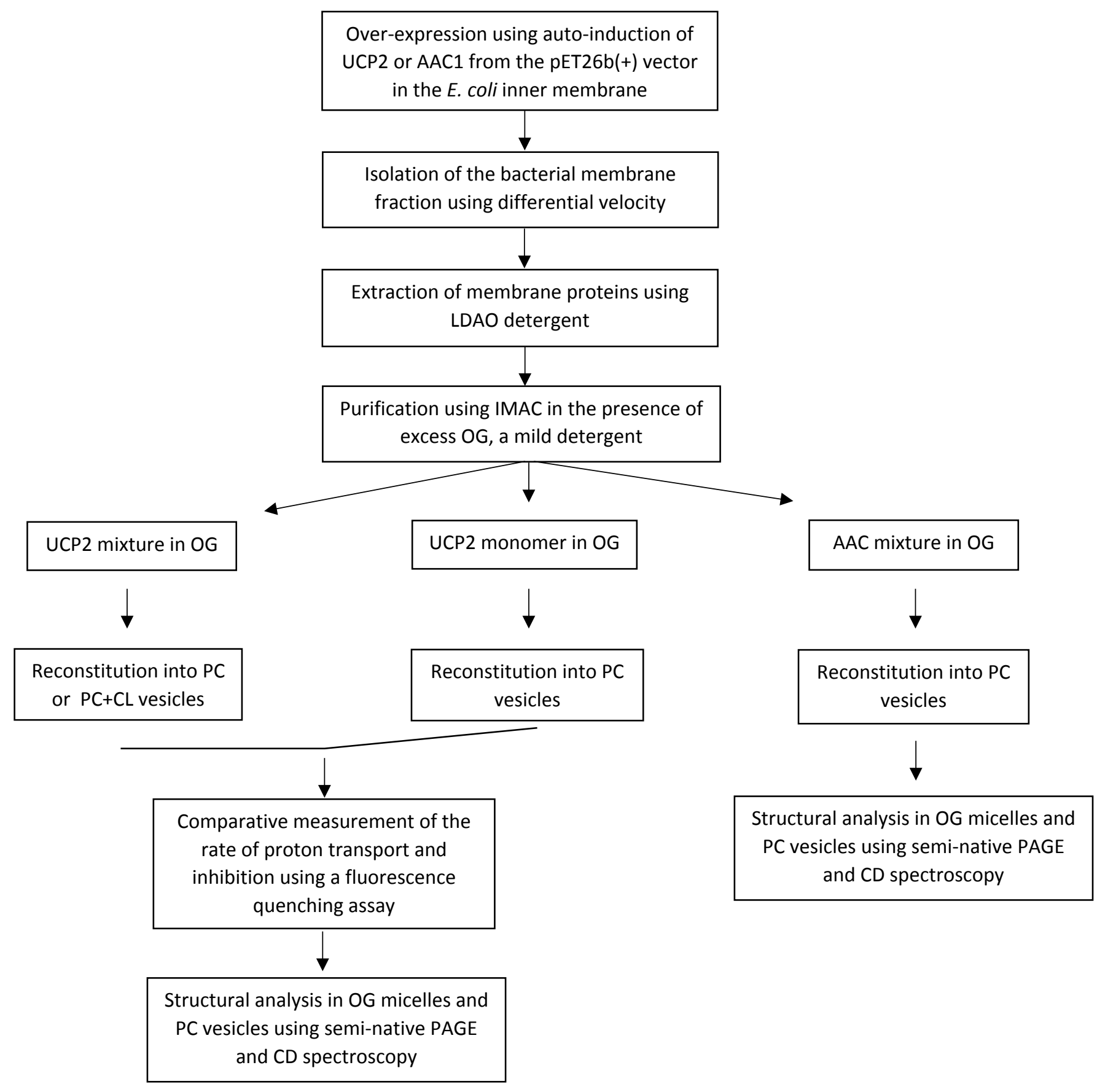

Scheme S1. Schematic representation of experimental procedures. 
Table S1. Salt-bridges formed in the oligomeric UCP2 systems. ${ }^{a, b}$

\begin{tabular}{|c|c|c|}
\hline \multirow{4}{*}{$\begin{array}{l}\bar{\Phi} \\
\stackrel{\Xi}{\Xi}\end{array}$} & \multicolumn{2}{|c|}{ Chain $1 \cdots$ Chain 2} \\
\hline & K109 & D202 \\
\hline & E112 & K206 \\
\hline & K164 & E46 \\
\hline \multirow{6}{*}{ 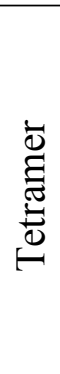 } & \multicolumn{2}{|c|}{ Chain $1 \cdots$ Chain 2} \\
\hline & K109 & D202 \\
\hline & K164 & E264 \\
\hline & \multicolumn{2}{|c|}{ Chain $3 \cdots$ Chain 4} \\
\hline & K104 & D202 \\
\hline & K164 & E264 \\
\hline
\end{tabular}

a Chains 1, 2, 3 and 4 correspond to the blue, red, orange, and grey monomeric units shown in Figure S6, respectively. ${ }^{b}$ Salt-bridges are ordered based on the number of subunit 1 (or 3 ) amino acids involved.

(a)

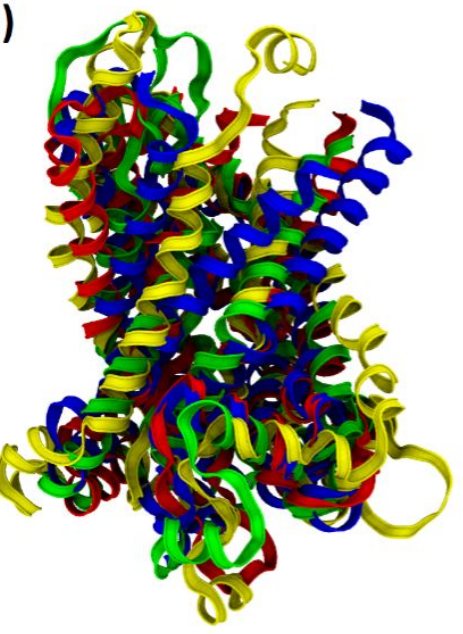

(b)

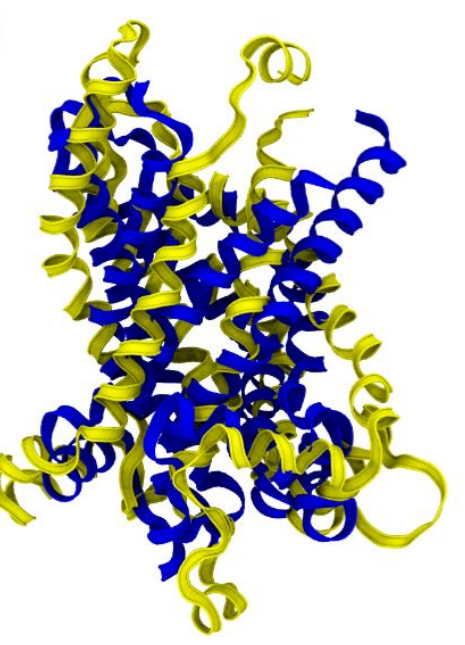

(c)

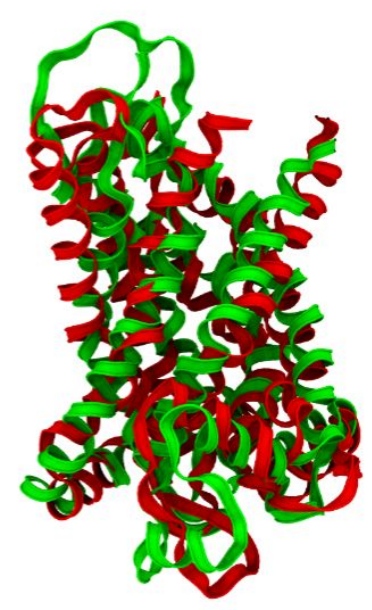

Figure S1. Overlay of initial and final structures: (a) AAC1 (PDB ID: 1OKC; blue), simulated UCP2 (PDB ID: 2LCK; yellow) starting from the NMR structure (350 ns, green), and simulated UCP2 made by homology modeling (350 ns, red). (b) AAC1 (PDB ID: 1OKC; blue), UCP2 (PDB ID: 2LCK; yellow). (c) Simulated NMR structure (green), and simulated UCP2 made by homology modeling using Swiss-Model ${ }^{1}$ (red). As seen, despite relative differences in 1OKC and 2LCK structures, the MD simulation based on these two initial structures (b) lead to comparable results (c). See also Figure S11. 

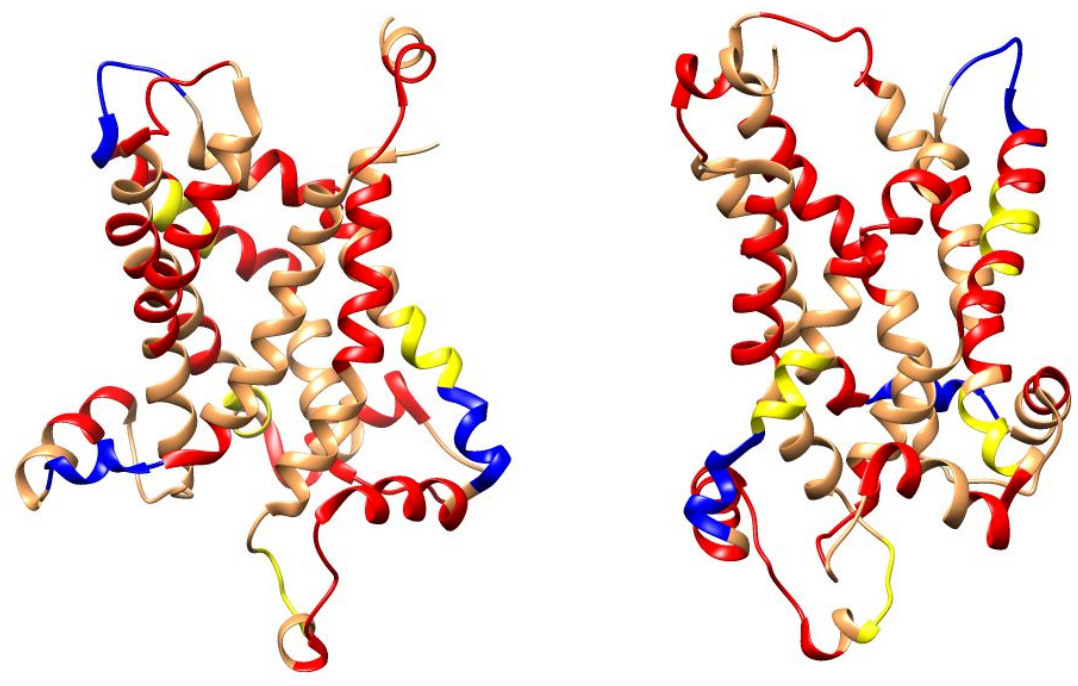

Figure S2. The two interacting faces of UCP2 selected to form a dimer (see also Figure S3). The GXXXG, GXXXLXXG, and SmXXXSm (Sm = Gly, Ala, Ser, Thr) motifs, which promote oligomerization are shown in yellow, blue, and red, respectively. The selected face includes all three motifs.

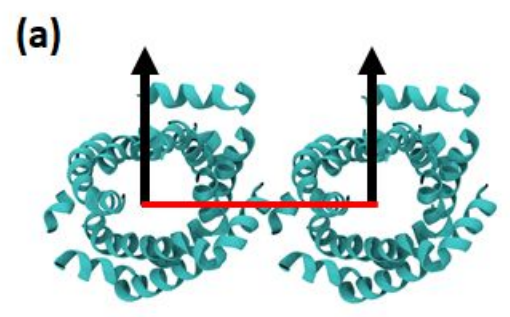

(b)

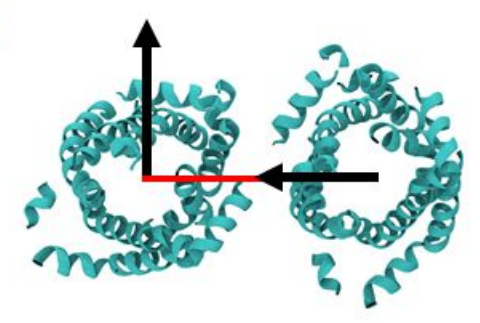

(c)

(d)
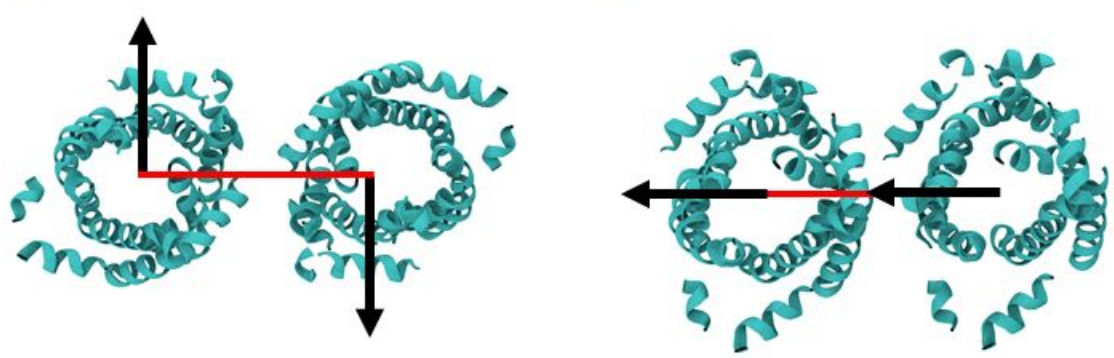

Figure S3. Four relative orientations of monomers to form a dimer. For clarity only helices are shown. Orientation (a) with maximal hydrophobic and electrostatic interactions minimal steric clashes was used in the simulations. This orientation allows for the oligomerization promoting sequence to have interactions in both dimeric and tetrameric forms. 


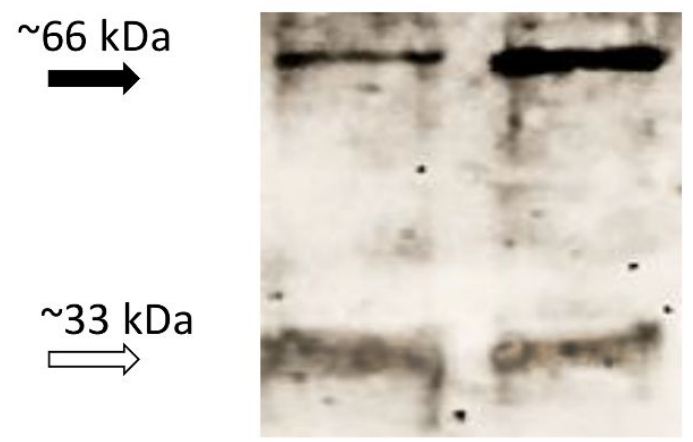

Figure S4. Western blot analysis of AAC1 probed with $\alpha$-AAC1 antibody confirms the copresence of monomeric and dimeric states in OG.
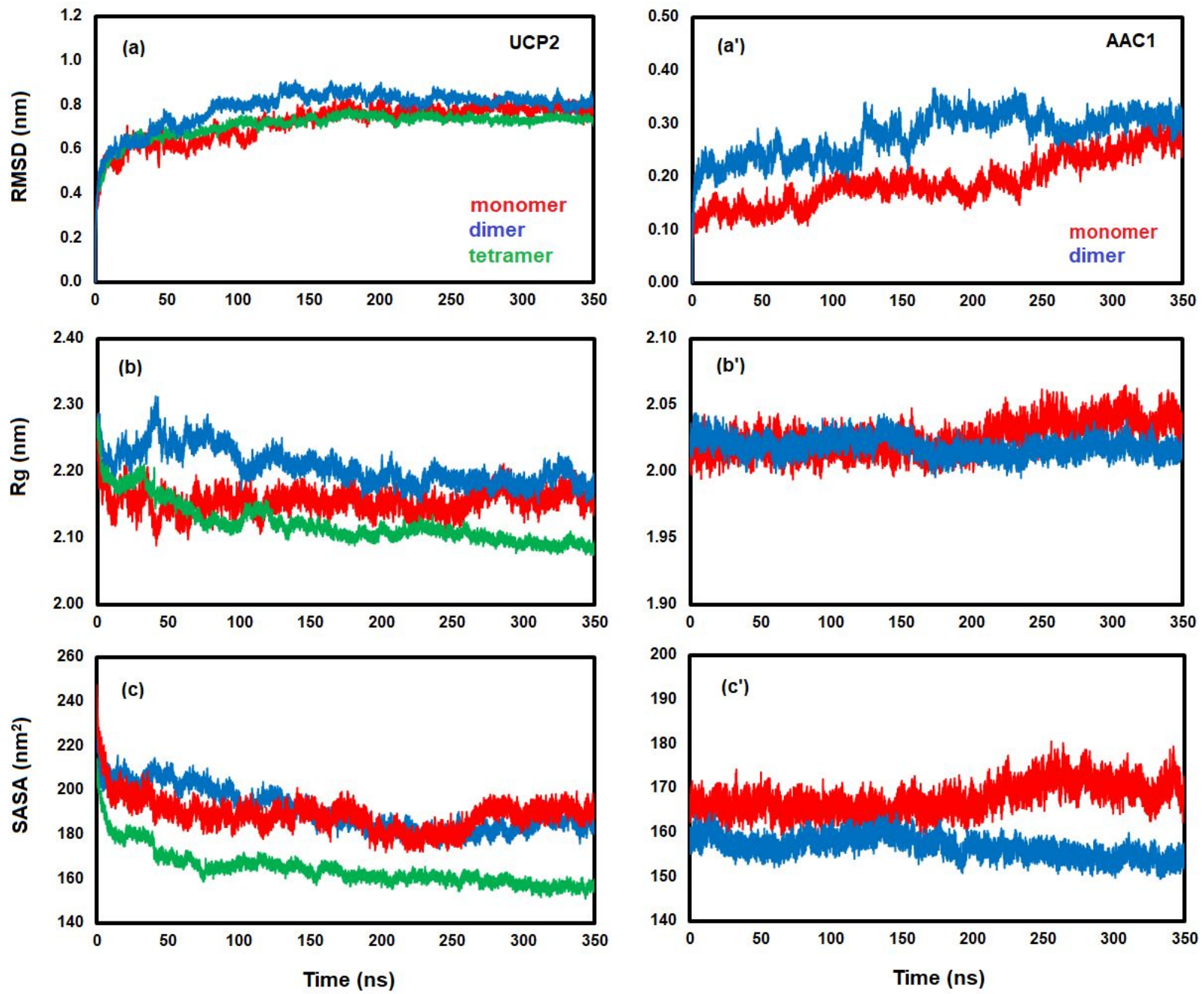

Figure S5. $\left(a-a^{\prime}\right)$ Protein backbone RMSD, $\left(b-b^{\prime}\right)$ radius of gyration $\left(R_{g}\right)$, and $\left(c-c^{\prime}\right)$ normalized solvent accessible surface area (SASA) for UCP2 and AAC1 in phospholipid bilayer membrane over the course of MD simulation. 
(a)

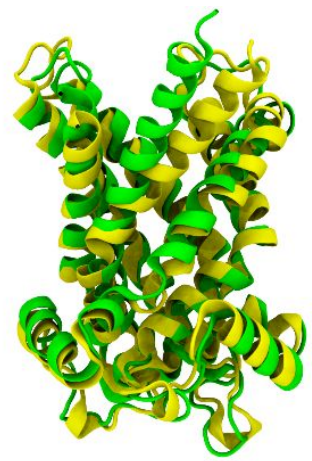

(d)

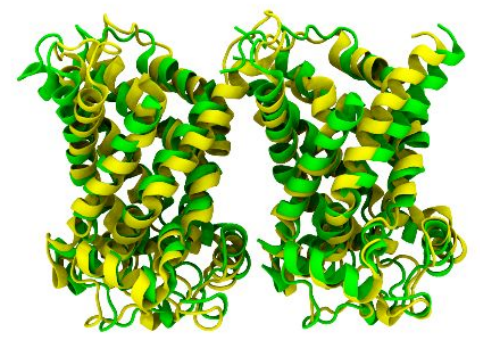

(b)

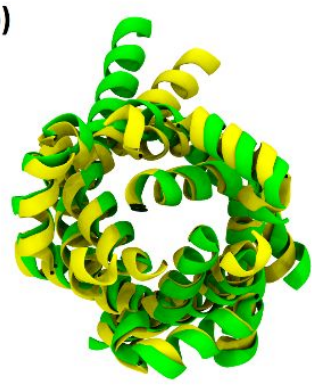

(e)

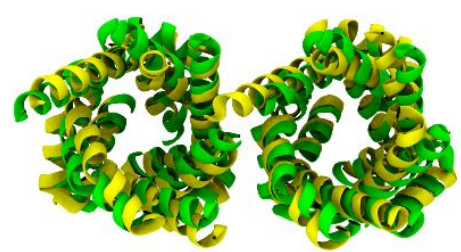

(c)

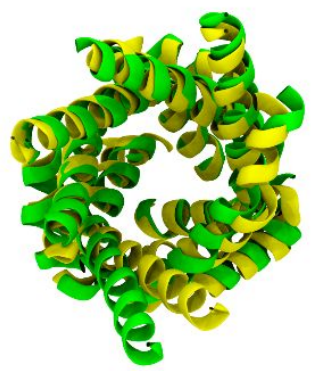

(f)

Figure S6. Overlay of the AAC1 monomer (a-c) and dimer (d-f) initial (PDB ID: 1OKC, yellow) and final (350 ns, green) structures. For clarity, only helices are shown in the top-view structures. Relatively small deviations from the initial structure are obvious for some of the TM helices.
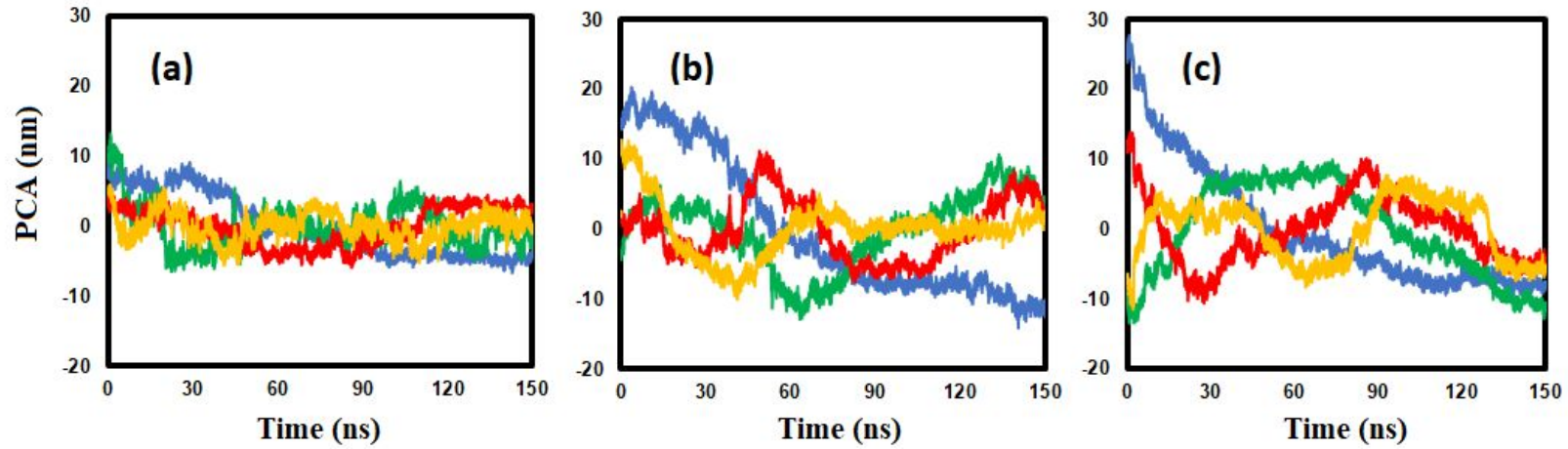

Figure S7. Projection of the first $150 \mathrm{~ns}$ MD simulation trajectories on the first 4 ( $1^{\text {st }}($ blue $), 2^{\text {nd }}$ (green), $3^{\text {rd }}$ (red), $4^{\text {th }}$ (yellow)) eigenvectors (principal component, PC) for (a) monomeric, (b) dimeric, and (c) tetrameric UCP2 in POPC bilayer. 
(a)

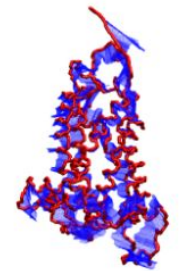

(a')

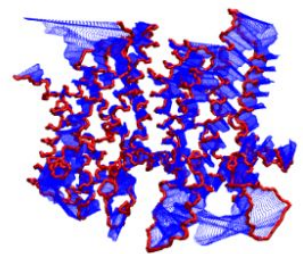

(a")

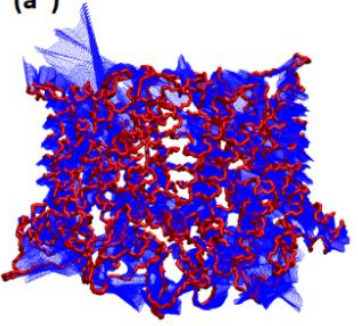

(b)

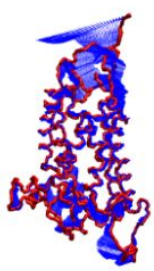

(b')

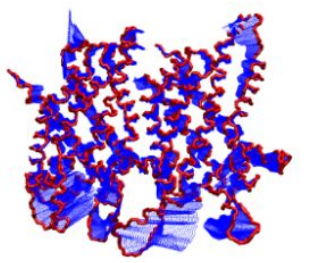

(b")

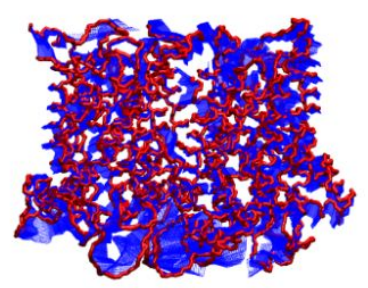

(c)

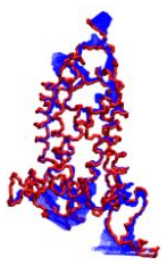

(c')

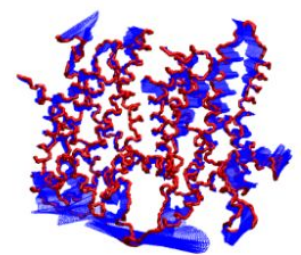

(c")

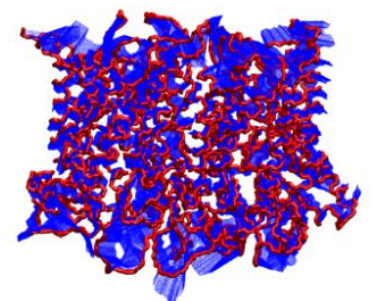

(d)

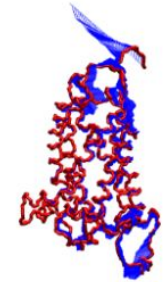

(d')

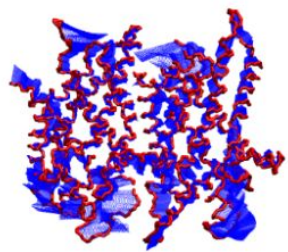

(d")

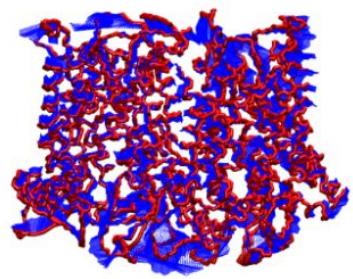

Figure S8. Visualization of PCA: Projections of the first $150 \mathrm{~ns}$ MD simulations on the first four eigenvectors for the monomeric $(a-d)$, dimeric $\left(a^{\prime}-d^{\prime}\right)$, and tetrameric $\left(a^{\prime \prime}-d^{\prime \prime}\right)$. Blue hatching represents the direction of motions.
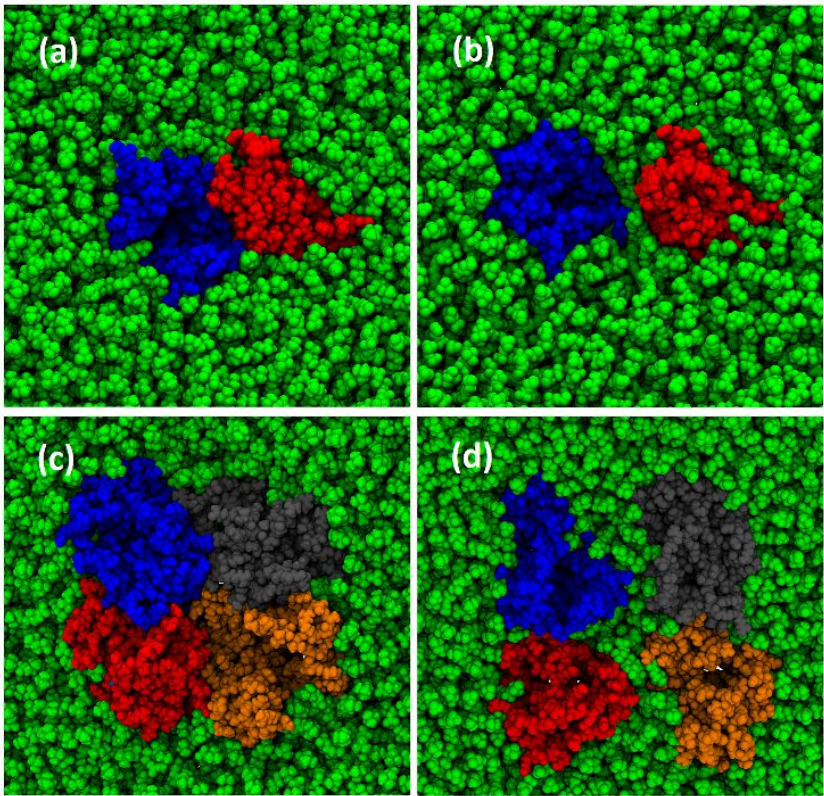

Figure S9. Initial and final structures of the dimeric (a-b) and tetrameric (c-d) UCP2 used for the dissociation process. Dissociation of dimer to its constituting monomers needs higher energy (18.5 kcal more) than dissociation of tetramer to its constituting dimers, suggesting tighter packing of monomers within the dimer compared to the two dimers within the tetramer. Chains 1,2, 3 and 4 are shown in blue, red, orange and grey, respectively. 


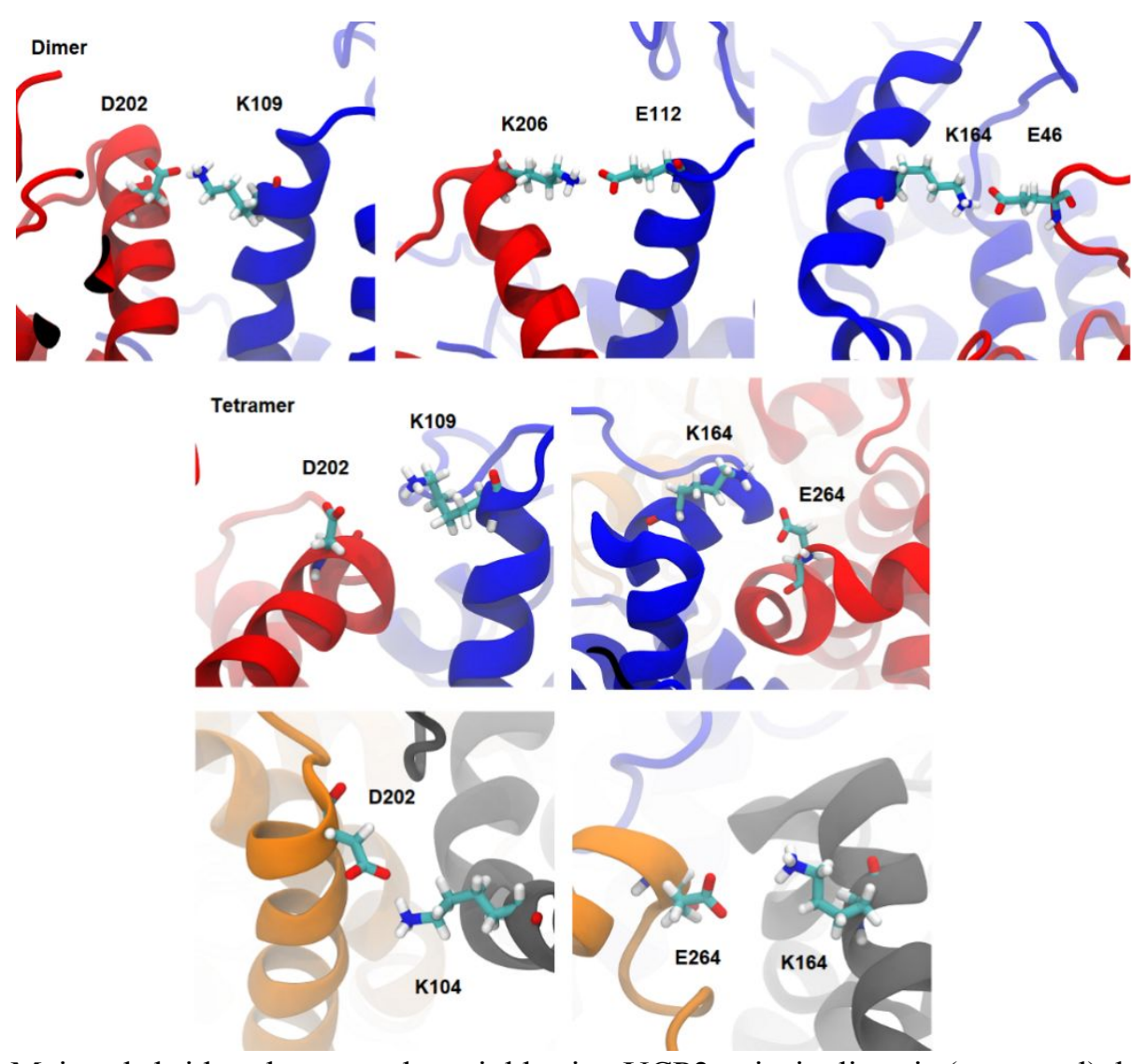

Figure S10. Main salt-bridges between the neighboring UCP2 units in dimeric (top panel), between chains 1 and 2 (middle panel), and chains 3 and 4 (bottom panel) in the tetrameric form. Chains 1, 2, 3, and 4 are shown in blue, red, grey, and orange, respectively. A salt bridge is formed when two amino acid with opposite charge interact within a distance of $<4.0 \AA .^{2,3}$ Accordingly, a $4.0 \AA$ cutoff was used to monitor osmotic permeability the formation of a salt bridge. 

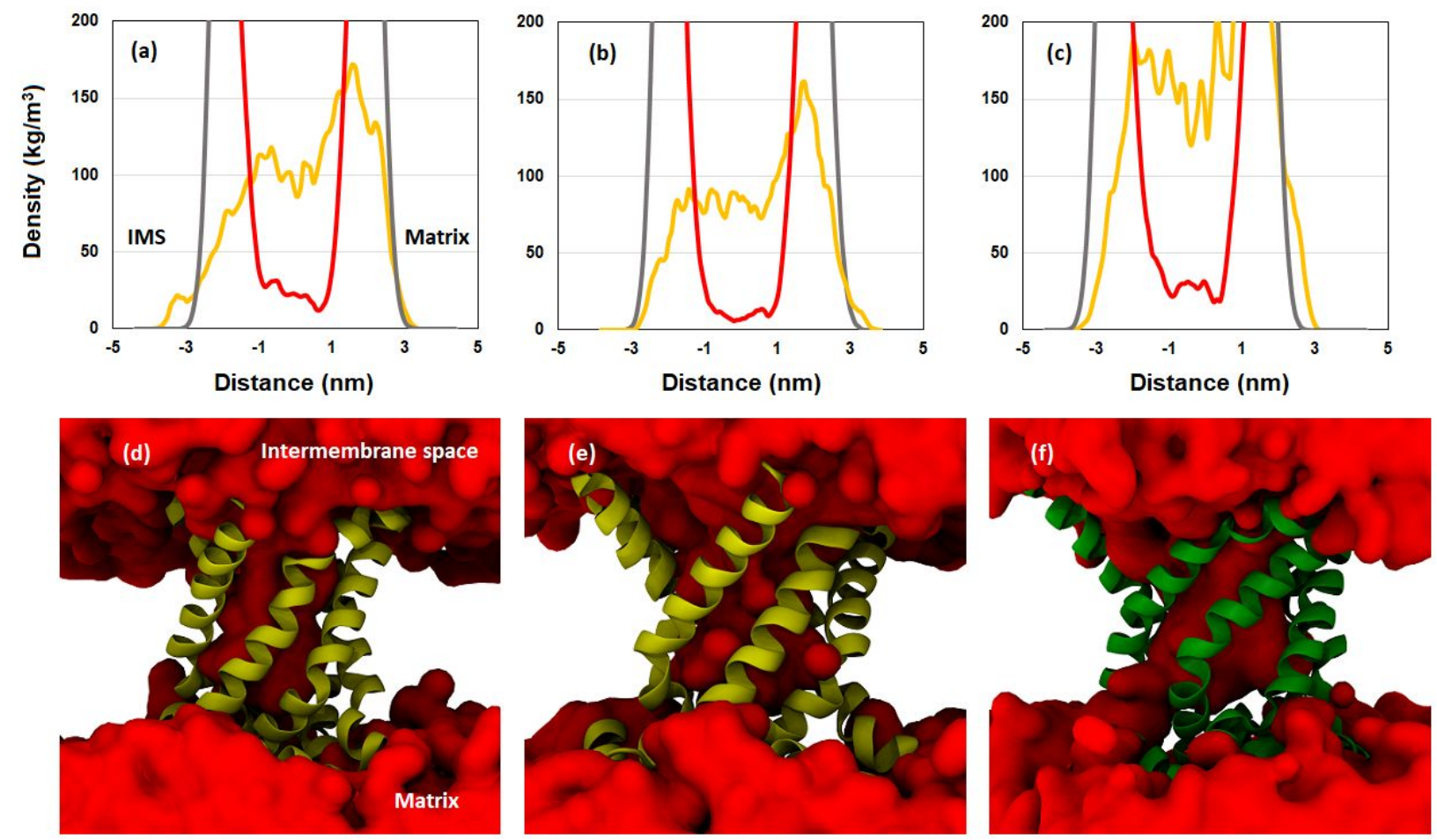

Figure S11. Density profile of protein (orange), water (red), and bilayer (grey) in simulated (a) UCP2 from 2LCK, (b) UCP2 modelled based on AAC1 structure (homology modelling), and (c) AAC1 monomeric systems and their corresponding structures. Snapshots $\mathrm{d}-\mathrm{f}$ : water: red, UCP2: yellow, and AAC1: green. Note that there is a small water density in all three cases. The only direct comparison is the AAC1 water density in DOPC bilayer by Škulj et al. which (the cytoplasmic open state with the matrix side more restricted) is in excellent agreement both qualitatively and quantitatively. ${ }^{4}$ The minimum water density is $11.7,6.7$, and $18.1 \mathrm{~kg} / \mathrm{m}^{3}$ for (a), (b), and (c), respectively. In terms of number density (as used by Škulj et al. and some other authors), they correspond to $0.39,0.22$ and $0.61 \mathrm{~nm}^{-3}$. The relation between mass density $(\rho)$ number density $(n)$ is given by $n=\rho \times N_{A} / M$, where $N_{A}$ is Avogadro's constant $\left(6.022 \times 10^{23} \mathrm{~mol}^{-1}\right)$ and $M$ is the molar mass $\left(18.015 \times 10^{-3} \mathrm{~kg} / \mathrm{mol}\right.$ for water $)$. 

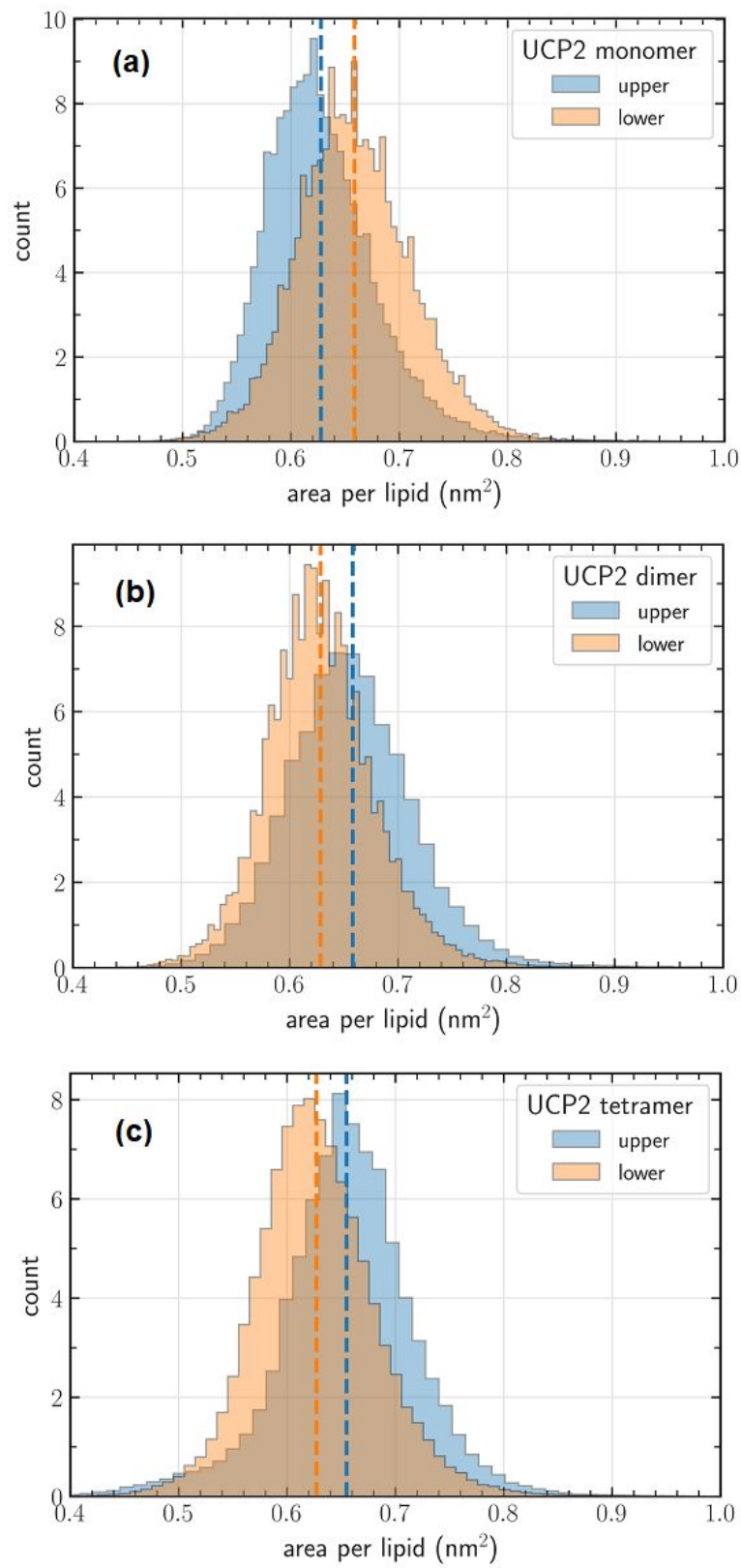

Figure S12. The area per lipid distribution in upper and lower leaflets in the cases of (a) monomeric, (b) dimeric, and (c) tetrameric UCP2. Average values are shown with dashed lines. As is seen, there is clear change in the distribution in both leaflets upon oligomerization of UCP2. 

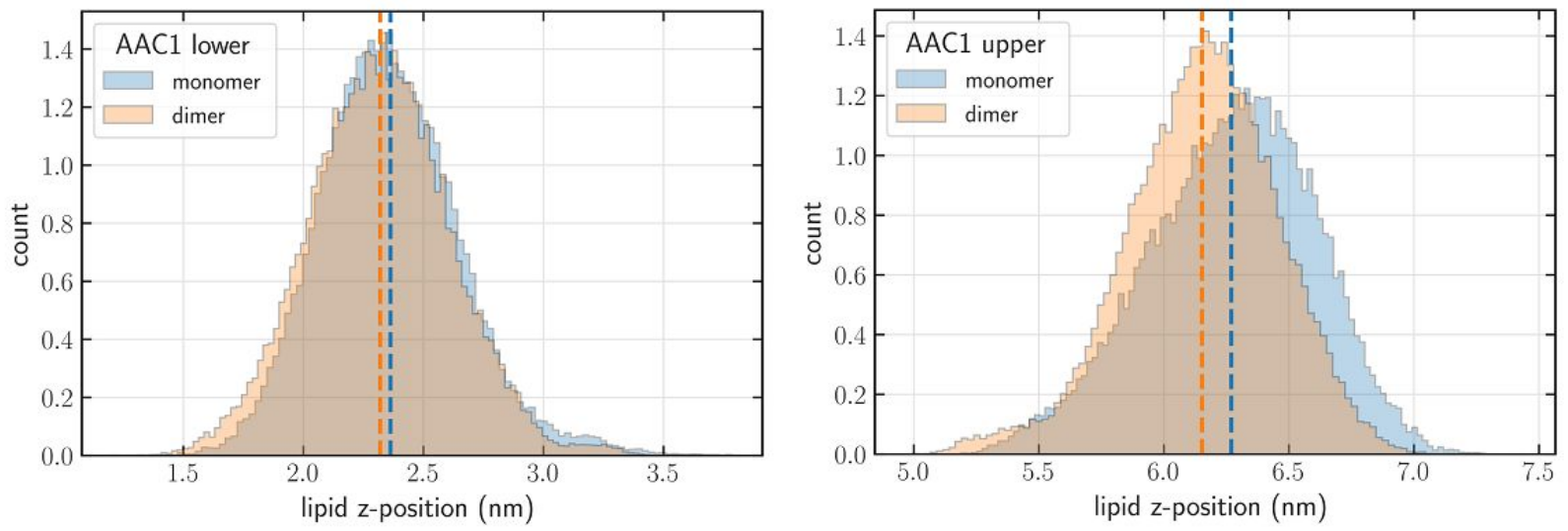

Figure S13. Distribution of the lipid head groups (phosphate) in lower (left, matrix side) and upper (right; cytoplasmic side) leaflets for monomeric and dimeric AAC1. As is seen, the distribution is different depending on the leaflet and oligomerization state of the protein.

\section{References}

1. Waterhouse, A., Bertoni, M., Bienert, S., Studer, G., Tauriello, G., Gumienny, R., Heer, F.T., de Beer, T.A.P., Rempfer, C., Bordoli, L., Lepore, R., Schwede, T. SWISS-MODEL: homology modelling of protein structures and complexes. Nucleic Acids Res. 2018, 46, 296303.

2. Donald, J. E., Kulp, D. W., DeGrado, W. F. Salt bridges: Geometrically specific, designable interactions. Proteins: Struct. Funct. Bioinforma. 2011, 79, 898-915.

3. Bosshard, H. R., Marti, D. N. \& Jelesarov, I. Protein stabilization by salt bridges: Concepts, experimental approaches and clarification of some misunderstandings. J. Mol. Recognit. 2004, $17,1-16$.

4. Škulj, S., Brkljaca, Z., Vazdar, M. Molecular dynamics simulations of the elusive matrixopen state of mitochondrial ADP/ATP carrier. Isr. J. Chem. 2020, 60, 735-743. 